\title{
THE SURFACE GRAVITIES OF Be STARS
}

\author{
GERALDINE J. PETERS
}

Dept. of Astronomy, University of California, Los Angeles, Calif., U.S.A.

\begin{abstract}
In an attempt to shed some light on the origin of the material in the envelopes of Be stars, surface gravities were determined for 30 objects by comparing their observed profiles of $\mathrm{H} \gamma$ and $\mathrm{H} \delta$ with those computed from the Princeton model atmospheres and the VCS theory of hydrogen line broadening. The program stars are predominately well-known Be stars and display a wide range of envelope spectra and $v \sin i$. The mean and range in $\log g$ for the Be stars appear to be identical to that obtained from a similar analysis on non-Be stars. No correlation was found between $\log \mathrm{g}$ and $\mathrm{H} \alpha$ emission strength or the strength and/or presence of emission of $\mathrm{Fe}$ II, $\mathrm{O}_{\text {I }} \lambda 7774 \AA$, or the infrared $\mathrm{Ca}$ II triplet. The suggestion made by Schild (1973) and Schild et al. (1974) that the extreme Be stars are in the post main sequence phase of rapid core contraction is weakened by the fact that there are several members of the class which have $\log g \geq 3.8$. All shell stars considered in the program appear to have low values of $\log g(\leq 3.5)$. Some possible explanations for this occurrence are discussed.
\end{abstract}

\section{Introduction}

One approach toward gaining an understanding of the origin of the material in $\mathrm{Be}$ star envelopes is to quantitatively study the spectra of the underlying stars. In particular, the surface gravity is a useful physical parameter which can help us establish to what extent the Be phenomenon is a normal stage in the post main sequence evolution of rapidly rotating B stars. Accordingly, I have obtained surface gravities for $30 \mathrm{Be}$ and $\mathrm{Be}$-shell stars by comparing the observed wings of their $\mathrm{Hr}$ and $\mathrm{H} \delta$ profiles with those computed from the Princeton model atmospheres and the hydrogen line broadening theory of Vidal et al. (1973). The program stars range from B0-B7 and display a wide range in $v \sin i$ and envelope spectra. Included in the sample are many objects which have been classified by Schild (1973) and Schild et al. (1974) as extreme Be stars.

The spectrograms used in the analysis were obtained with the coudé spectrograph of the 120 -in $(305 \mathrm{~cm})$ telescope at Lick Observatory and ranged in dispersion from 5.5-16 $\AA \mathrm{mm}^{-1}$. Two-thirds of the objects were observed at the higher dispersion and more than one plate was available for over one-third of the stars.

\section{Predictions}

What do competing theories for the origin of Be envelopes predict for the value of $\log g$ of the underlying star? Three theories which have been recently considered along with the range in $\log g$ which they suggest are briefly discussed below.

\section{1. 'RotATIONALLY UNSTABLE' SINGLE STARS}

Computations made by several investigators have suggested that rapidly rotating B-type stars will reach a point of critical rotation at some stage during their post main 
sequence evolution (Sackmann and Anand, 1970; Crampin and Hoyle, 1960). The details of the star's subsequent behavior remain uncertain and a source of controversy. Some researchers feel that when the star reaches this stage, 'rotationally forced ejection' of material via magnetic coupling of envelope to star can occur (Limber and Marlborough, 1968; and Limber, 1970). However, it should be stated that alternatives to the latter idea have been presented (Bodenheimer and Ostriker, 1973 and other papers by these authors referenced therein). Schild (1973) and Schild et al. (1974) claim that one can spectroscopically isolate a group of Be stars, called extreme Be stars, which are losing mass during the post main sequence phase of rapid core contraction as a result of an instability caused by rapid rotation. These Be stars have strong, permanent $\mathrm{H} \alpha$ emission, Fe II emission, and infrared excesses. If Schild et al. are correct, then stars classified as extreme Be stars should have a fairly narrow range in $\log g, 3.5 \leqslant \log g \leqslant 3.7$.

\subsection{Mass-eXChange BinaRies}

Recently, several researchers have suggested that at least some Be stars gain their emission line envelopes through binary mass exchange (Plavec, 1970; Plavec, 1973; Peters and Polidan, 1973; Kriz and Harmanec, 1975). In fact, later in this symposium arguments will be presented by Dr P. Harmanec that all Be stars may be interacting binary stars. The idea of binary mass exchange as an explanation for the existence of Be stars is attractive since it can explain, at least qualitatively, not only the source of the material in $\mathrm{Be}$ envelopes but also the high values of $v \sin i$ associated with $\mathrm{Be}$ stars. The expected range in $\log g$ for interacting binary $\mathrm{Be}$ stars is large, $3.3 \leqslant$ $\log g \leqslant 4.0$, and uncertain on account of our present lack of understanding of the details of mass accretion onto the transformed primary.

\subsection{Pre-main sequence objects}

We must consider the possibility that some stars classified as 'classical Be stars' may actually be pre-main sequence objects which have not completely lost or accreted their 'cocoons'. Prime candidates for such a class of objects would be located in young $\mathrm{OB}$ associations such as $\mathrm{h}$ and $\chi$ Per and the Scorpio-Centaurus association. Lack of nebulosity in the vicinity of such objects may confuse one as to the star's evolutionary state. At this time it is uncertain just how long it will take a rapidly rotating pre-main sequence B-type star to accrete and/or dissipate material in a cocoon. The suspected values of $\log g$ for these objects are uncertain; however, we suggest the following range: $3.0 \leqslant \log g \leqslant 3.6$.

\section{Assignment of Effective Temperatures}

The hydrogen line profiles in early B-type stars are a function both of gravity and temperature. The amount of narrowing of a profile due to a change in log $g$ of 0.1 is nearly identical to that resulting from a change in effective temperature of $1000 \mathrm{~K}$. Therefore, it is essential that one obtain a fairly good estimate of the effective temperature of a B-type star before attempting to determine a surface gravity from a theoretical profile grid. 
For this investigation, values of $T_{\text {eff }}$ were assigned to the program stars on the basis of : (1) spectral types, (2) $U B V$ colors, and (3) photospheric line strengths and ratios. For most stars, the spectral types determined by Lesh (1968) were adopted. The $U B V$ colors for the program stars were obtained by taking weighted means of values listed in the compilation of Blanco et al. (1968). Colors were corrected for interstellar reddening using the two color relationship of Johnson (1966). Temperatures were derived from the writer's calibrations of spectral type and $(U-B)_{0}$ vs $T_{\text {eff }}$ (Peters, 1976). Although continuous Balmer emission (or absorption) contributes significantly to the observed colors in some cases, it was found that, in general, the temperature indicated by the corrected $(U-B)_{0}$ color was in close agreement with the one suggested by the spectral type.

The preferred method of assigning a temperature to a $\mathrm{Be}$ star is to perform a detailed study of the star's spectrum using model atmospheres to interpret the individual line strengths. An investigation of this nature is presently in progress for several of the program stars (i.e. $v$ Cyg, $31 \mathrm{Peg}, \chi$ Oph, $\mu$ Cen, HR 2142, and $\phi$ Per).

\section{Analysis of the $\mathrm{H} \boldsymbol{\gamma}$ and $\mathrm{H} \delta$ Profiles}

In interpreting the hydrogen line profiles observed in Be stars, we are faced with some additional complications which are not present in those profiles observed in sharp-lined, early B-type stars. Some problems include emission line contamination of the profile, rotational broadening, and broadening due to electron scattering in the star's envelope. In this investigation, I have concluded that the problems are indeed surmountable and that the most useful portion of the hydrogen line profile for analysis is $7<\Delta \lambda<14 \AA$. For stars in which the effective temperature can be determined to within $1000 \mathrm{~K}$, the uncertainty in $\log g$ obtained by the adopted procedure is less than 0.2 .

For the majority of the program stars, emission altered the hydrogen line profile only in the vicinity of the line center. Only in the case of HR 2855 was there any evidence of emission line contamination at a greater distance than $7 \AA$ from the center of the line. Some individual cases will be discussed toward the end of this section.

Rotational broadening has a very small effect on the wing portion of a hydrogen line profile at distances greater than $7 \AA$ from the line center. For a star with $v \sin i>300 \mathrm{~km} \mathrm{~s}^{-1}$, one would obtain an apparent $\log g$ which is about 0.1 too high upon using an 'unrotated' grid of theoretical profiles. Burbidge and Burbidge (1953) also noted that moderate amounts of rotation do not affect the wings of hydrogen line profiles.

In their detailed study of the envelope spectra of six Be stars, Burbidge and Burbidge (1953) presented evidence that the wings of the hydrogen lines in Be stars are significantly broadened by electron scattering in the circumstellar envelopes. They obtained optical depths up to 0.4 due to this process. However, the present investigation does not support their conclusion even though four stars were common to both programs (11 Cam, $48 \mathrm{Per}, \omega \mathrm{CMa}$, and $\beta$ Psc). Whereas Burbidge and Burbidge compared the Be stars' hydrogen line profiles to the profiles observed in two standard stars ( $\zeta$ Cas and $\eta$ Aur), the $\mathrm{H} \gamma$ and $\mathrm{H} \delta$ profiles observed in the Be stars 
considered in this program were compared to a theoretical grid of profiles which have been used successfully to obtain surface gravities for ten normal, sharp-lined, early B-type stars (Peters 1976). The temperature of $\zeta$ Cas (about $24500 \mathrm{~K}$ ) is significantly higher than the temperatures of the four stars common to the Burbridge program and this investigation. The temperature of $\eta$ Aur is comparable to that of 48 Per, but Burbidge and Burbidge compared this star to $\zeta$ Cas. The fact that the temperatures of the standard stars used by Burbidge and Burbidge were not representative of their program stars is most likely the reason for their conclusion regarding the importance of electron scattering in the envelope as a source of broadening in the wings of hydrogen lines.

The surface gravities which were obtained for the Be and Be-shell stars considered in this program are tabulated in Tables I and II. The value quoted for $\log g$ is a mean

TABLE I

Summary of surface gravities for Be stars

\begin{tabular}{lllll}
\hline Star & Sp. type ${ }^{\mathrm{a}}$ & $v \sin i^{\mathrm{b}}$ & $T_{\text {eff }}$ & $\log g$ \\
\hline HD 7636 & B2 & $-\mathrm{km} \mathrm{s}^{-1}$ & $20000 \mathrm{~K}$ & 4.0 \\
$\phi$ Per & B0 & 450 & 28000 & 4.0 \\
48 Per & B3 & 217 & 16000 & 3.5 \\
11 Cam & B2.5 & 131 & 19000 & 4.0 \\
HR 2142 & B1 & 350 & 25500 & 3.9 \\
$\omega$ CMa & B2 & 137 & 20000 & 3.5 \\
HR 2825 & B3 & 33 & 17000 & 3.7 \\
HR 2855 & B0.5 & 244 & 28000 & 4.0 \\
$\mu$ Cen & B2 & 191 & 20000 & 3.5 \\
Oph & B1.5 & 123 & 24000 & 3.8 \\
66 Oph & B2 & 241 & 23000 & 4.0 \\
HR 7249 & B2 & 226 & 22000 & 3.6 \\
25 Cyg & B3 & 229 & 16500 & 3.3 \\
28 Cyg & B2.5 & 310 & 20000 & 3.7 \\
25 Vul & B7 & 250 & 13000 & 3.3 \\
59 Cyg & B1.5 & - & 25000 & 4.0 \\
$v$ Cyg & B1.5 & 261 & 24000 & 3.8 \\
6 Cep & B3 & 148 & 17500 & 3.5 \\
16 Peg & B3 & 152 & 17000 & 3.5 \\
31 Peg & B2 & 134 & 20000 & 3.5 \\
$\pi$ Aqr & B1 & 278 & 27000 & 3.9 \\
$\beta$ Psc & B6 & 147 & 14500 & 3.5 \\
\hline
\end{tabular}

${ }^{a}$ From Lesh (1968); a few types are from the author.

${ }^{b}$ From Uesugi and Fukuda (1970); value for HR 2142 from Peters (1972).

from $\mathrm{H} \gamma$ and $\mathrm{H} \delta$ and, in the cases where more than one plate was available for the star, the mean was determined by weighting the observations according to the dispersion and quality of the plate. The values of $\log g$ deduced from $\mathrm{H} \gamma$ and $\mathrm{H} \delta$ usually agreed within 0.1 . For stars in which variations of emission lines or shell components were observed, the wings of the hydrogen line profiles remained unchanged. A general discussion of the results is presented in the following section. Six individual stars will be discussed below. 
TABLE II

Summary of surface gravities for shell stars ${ }^{\mathrm{a}}$

\begin{tabular}{|c|c|c|c|c|}
\hline Star & Sp. type ${ }^{b}$ & $v \sin i^{\mathrm{c}}$ & $T_{\text {eff }}$ & $\log g$ \\
\hline $\begin{array}{l}\psi \text { Per } \\
28 \text { Tau } \\
\zeta \text { Tau } \\
\text { AX Mon } \\
\text { HD 173219 } \\
\varepsilon \text { Cap } \\
o \text { And } \\
\text { HD 218393 }\end{array}$ & $\begin{array}{l}\text { B5 } \\
\text { B7 } \\
\text { B1 } \\
\text { B1 } \\
\text { B0.5 } \\
\text { B2.5 } \\
\text { B6 } \\
\text { B2 }\end{array}$ & $\begin{array}{l}398 \mathrm{~km} \mathrm{~s}^{-1} \\
341 \\
310 \\
430 \\
- \\
274 \\
330 \\
-\end{array}$ & $\begin{array}{l}15500 \mathrm{~K} \\
13000 \\
25000 \\
25000 \\
27000 \\
19000 \\
15000 \\
22000\end{array}$ & $\begin{array}{l}3.3 \\
3.3 \\
3.5 \\
3.5 \\
3.5 \\
3.2 \\
3.2 \\
3.4\end{array}$ \\
\hline
\end{tabular}

${ }^{a}$ Stars which show 'metallic' shell lines in the ground-based portion of their spectra.

${ }^{b}$ From Lesh (1968) or author.

${ }^{c}$ From Uesugi and Fukuda (1970).

HR 2825 has the lowest projected rotational velocity of the stars considered in this program $\left(33 \mathrm{~km} \mathrm{~s}^{-1}\right)$ and, hence, the least emission line contamination in its hydrogen line profiles. As shown in Figure 1 for $\mathrm{H} \delta$, the emission is confined to $\pm 2 \AA$ from the center of the line while the remainder of the profile fits well to one computed from the Princeton model atmosphere of $T_{\text {eff }}=16800 \mathrm{~K}, \log g=3.5$. The photospheric parameters $\left(T_{\text {eff }}, \log g\right.$, and chemical composition) for HR 2825 appear to be quite

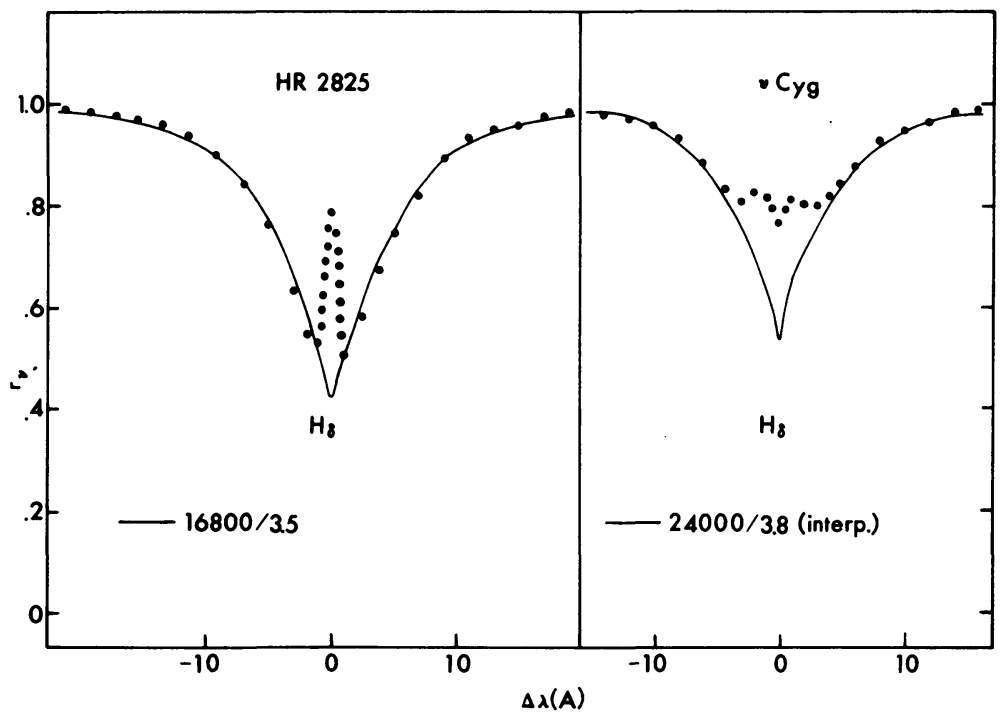

Fig. 1. Comparison between observed profiles of H $\delta$ in HR 2825 and $v$ Cyg and those computed from the Princeton model atmospheres. The model parameters are indicated below the profiles.

close to those for $\iota$ Her (Peters 1976). Kodaira and Scholz (1970) reached a similar conclusion from their analyses of these stars.

$v$ Cyg is a good example of a Be star with an intermediate value of $v \sin i$ $\left(\sim 250 \mathrm{~km} \mathrm{~s}^{-1}\right)$. The Balmer line emission appears to be constant in strength and 
profile and contributes only to the inner $\pm 5 \AA$ of the $\mathrm{H} \gamma$ and $\mathrm{H} \delta$ features. The wings of $\mathrm{H} \gamma$ and $\mathrm{H} \delta$ can be fit to interpolated profiles corresponding to a model of $T_{\text {eff }}=24000 \mathrm{~K}, \log g=3.8$ (see Figure 1).

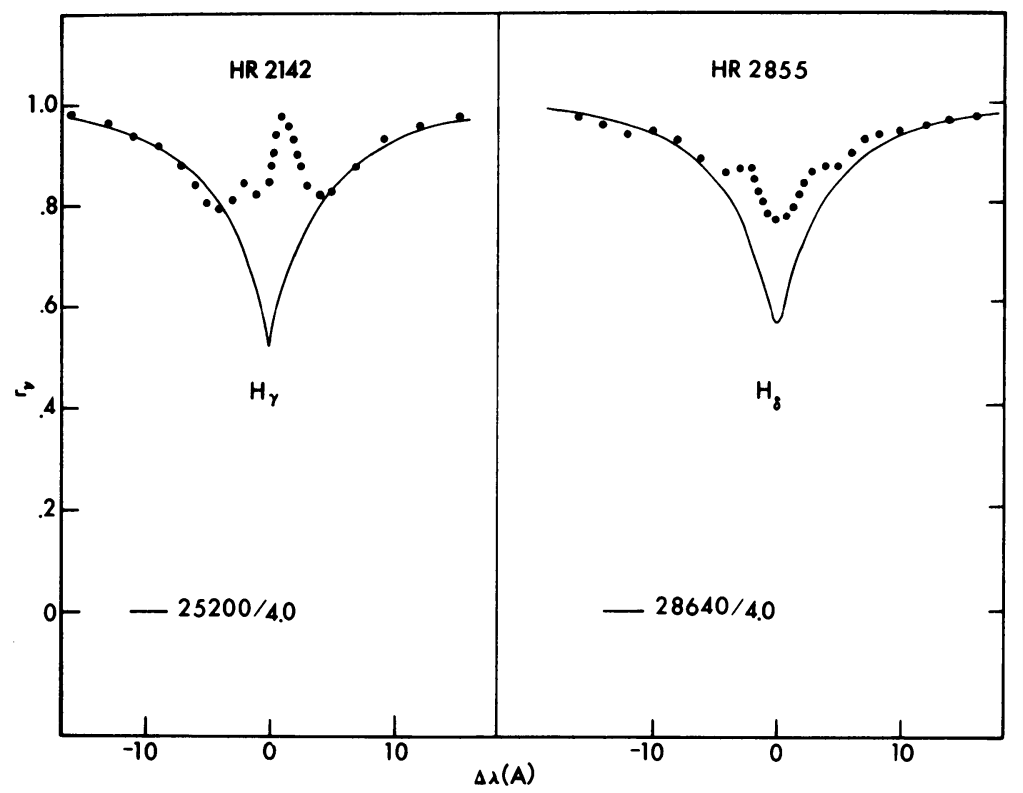

Fig. 2. Comparison between observed and computed hydrogen line profiles for HR $2142\left(\Phi_{s}=0.09\right)$ and HR 2855. The model parameters are indicated below the profiles.

Figure 2 shows that the observed profile of $\mathrm{H} \gamma$ in HR 2142 closely matches one computed from the Princeton model atmosphere of $25200 \mathrm{~K}, \log g=4.0$. The profiles of the Balmer lines in HR 2142 undergo cyclic variations ( $V / R$ type) with a period of 80.86 (Peters, 1972). The profile which appears in Figure 2 was observed at $\phi_{s}=0.09$ when $R \gg V$. Emission contributes at distances less than $7 \AA$ from the line center and the wings of the hydrogen line profiles remain constant with phase. The $v \sin i$ for HR 2142 is about $350 \mathrm{~km} \mathrm{~s}^{-1}$.

HR 2855 showed the highest degree of emission line contamination to its hydrogen line profiles. As one can see from Figure 2, there appears to be emission in $\mathrm{H} \delta$ at $9 \AA$ from the line center! This fact combined with the apparent high temperature and gravity produces a very low contrast hydrogen line profile; the most conspicuous feature is the weak, somewhat broad shell core. The uncertainties in $T_{\text {eff }}$ and $\log g$ for HR $2855\left(\Delta T_{\text {eff }}=2000 \mathrm{~K}, \Delta \log g=0.5\right)$ are considered to be the largest of all the program stars.

The program also included eight Be-shell stars. The values of $\log g$ which were obtained for the shell stars are tabulated in Table II. In this investigation, it was found that shell stars tend to have low gravities. Whereas the mean $\log g$ for the Be stars was 3.7 , the shell stars showed values of $\log g \leq 3.5$. In Figure 3 , we find the comparison between the observed $\mathrm{H} \delta$ profiles of two shell stars, $\zeta$ Tau and $o$ And, and computed profiles. 


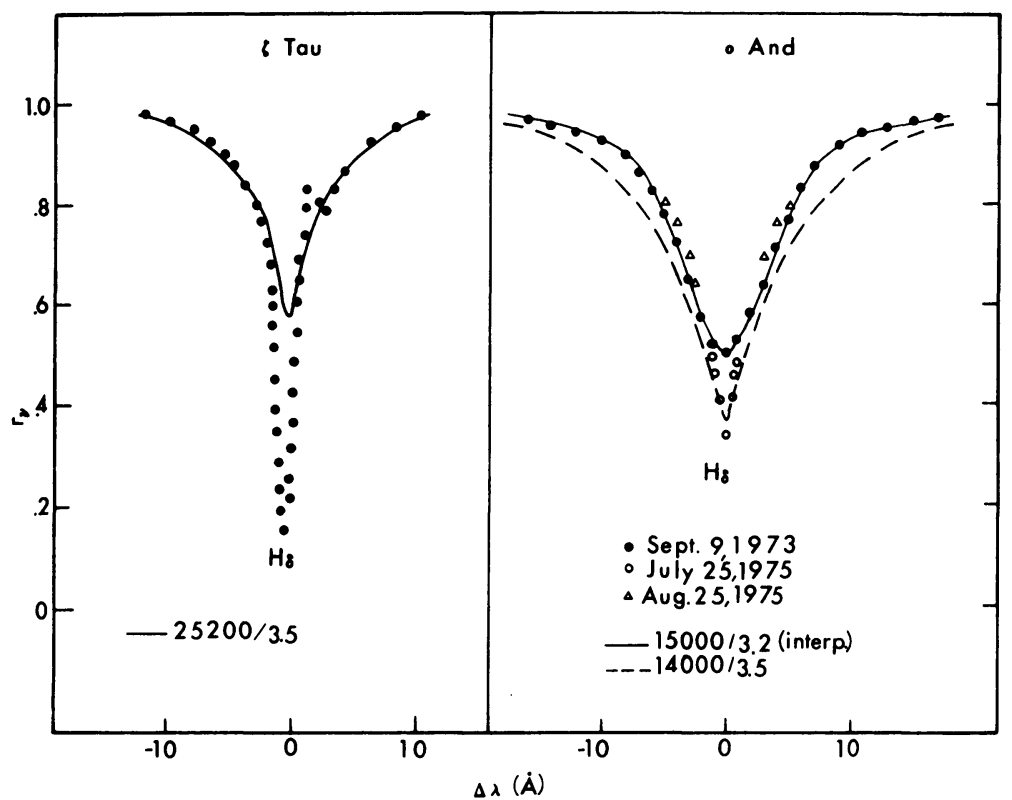

Fig. 3. Comparison between observed and computed $\mathrm{H} \delta$ profiles for $\zeta$ Tau (in 1974 and 1975) and $o$ And (in 1973 and 1975). The observations of $o$ And in 1975 were made during its recent shell phase.

This investigation suggested that the temperature of $\zeta$ Tau is close to $25000 \mathrm{~K}$ and $\log g$ is near 3.5. When transformations between systems of model atmospheres are considered, the latter atmospheric parameters appear to be in agreement with those determined by Heap (1975).

$o$ And is a rapidly rotating B6 star which has shown a recurrent shell phase (Slettebak, 1952). After remaining inactive for nearly two decades, $o$ And has recently developed another shell (Koubsky, 1975; Peters and Polidan, 1975). In Figure 3 , the profiles of $\mathrm{H} \delta$ observed outside of and during a shell phase are compared with those computed from the Princeton model atmospheres. Although the hydrogen line profiles observed during the shell phase show both shell type absorption and weak emission in the vicinity of the line center, the wings remain unchanged from their profiles observed outside of shell phase. One would obtain the same value of $\log g$ both inside and outside of shell phase. When the comparisons between the observed and computed profiles of $\mathrm{H} \gamma$ and $\mathrm{H} \delta$ are made, it becomes evident that the average surface gravity for $o$ And is quite low. If the effective temperature is near $15000 \mathrm{~K}$ (a value consistent with the star's spectral type), then $\log g$ is about 3.2. The apparent low gravities of shell stars will be further discussed in Section 5 of this paper.

\section{Discussion of Results}

It can be seen from the tabulated values of $\log g$ in Table I that the 'classical' Be stars display a wide range in surface gravity $(3.3 \leq \log g \leq 4.0)$. The mean value of $\log g$ for 
the Be stars considered in the program is 3.7. A similar analysis was performed for ten sharp-lined, non-emission B-type stars (Peters, 1976). The mean and range in $\log g$ was identical to the one obtained in this investigation. The program stars included in each investigation are predominantly field stars.

Can one find a spectroscopic feature whose strength and/or presence correlates with the value of $\log g$ of a Be star? Possible candidates are considered below.

In Figure 4, we present a plot of the value of $\log g$ vs the $\alpha$-index for the star. Both Be stars and shell stars are included. Values of the $\alpha$ index were obtained from

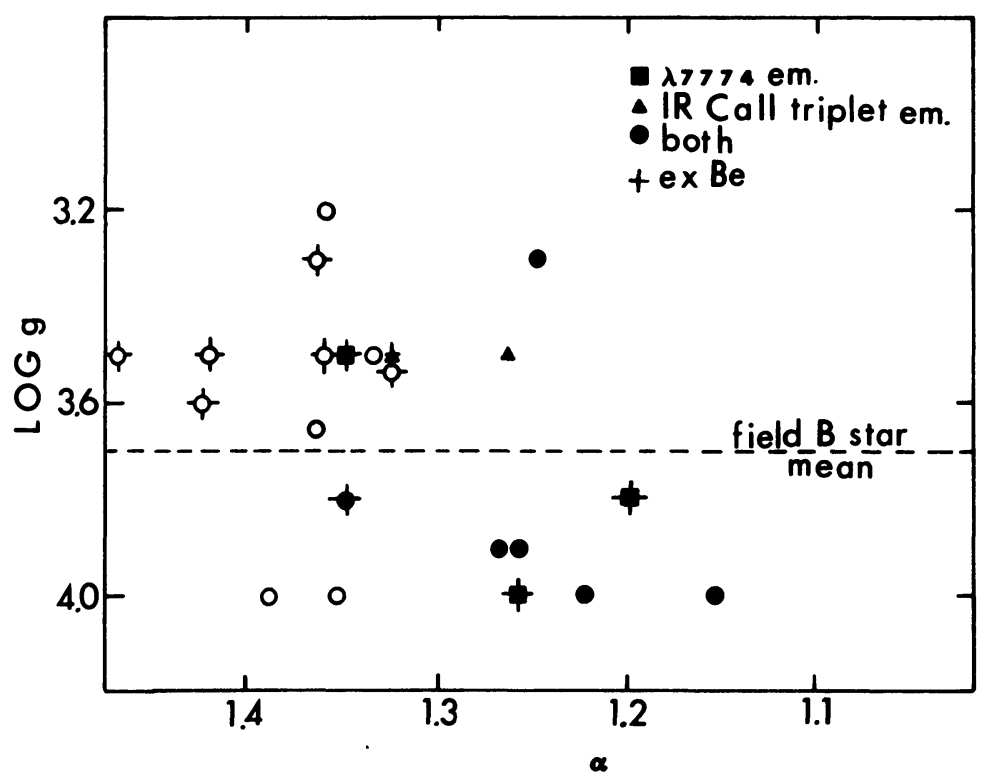

Fig. 4. The value of $\log g$ vs $\mathrm{H} \alpha$ emission line strength. Open circles represent objects which do not show O I $\lambda 7774 \AA$ emission or emission at the infrared Ca II triplet. Extreme Be stars are indicated by crosses superposed on the star's symbol. The dashed line denotes the mean value of $\log g$ for field, non-Be stars.

Feinstein (1974). Certainly, there is no evidence that the surface gravity of a Be star is correlated with the strength of the $\mathrm{H} \alpha$ emission. It is also evident from the plot in Figure 4 that there is no correlation between the value of $\log g$ and the presence of emission at $\lambda 7774 \AA$ of $\mathrm{O}_{\mathrm{I}}$ or the infrared $\mathrm{Ca}$ II triplet. In addition, the presence of $\mathrm{Fe}$ II emission [which correlates well with the presence of $\lambda 7774 \AA$ emission (Peters, 1974)] does not appear to be a function of $\log g$.

The stars classified as extreme Be stars by Schild (1973) and Schild et al. (1974) are noted in Figure 4 by crosses superposed on the stars' symbols which indicate whether emission is present at $\lambda \mathbf{7 7 7 4}$ or the infrared $\mathrm{Ca}$ II triplet. It can be seen that the extreme Be stars are not restricted to a narrow range in $\log g(3.5 \leq \log g \leq 3.7)$ which one would expect if every member of this class is in the post main sequence phase of rapid core contraction. Although several members of the group have values of $\log g$ close to 3.5 , there are certain extreme Be stars which definitely have $\log g \geq 3.8$ (i.e. $11 \mathrm{Cam}, v \mathrm{Cyg}$ ). We cannot dismiss entirely the possibility that some Be stars may 
indeed be losing mass due to an instability which may occur during the phase of rapid core contraction; however, it appears that such objects cannot be isolated by spectroscopic criteria alone. For an individual Be star which has an intermediate value of $\log g$ and for which there is no evidence of binary mass exchange or pre-main sequence contraction, at present one cannot determine the source of the material in its envelope.

This study has also revealed that there is no correlation between the surface gravity of the Be star and $v \sin i$. From the values of $v \sin i$ tabulated in Table $\mathrm{I}$, it can be seen that both low and high surface gravities characterize 'sharp-lined' stars (i.e. $\beta$ Psc and $11 \mathrm{Cam}$ ) and 'broad-lined' stars (i.e. $28 \mathrm{Cyg}$ and $\phi$ Per).

At the beginning of this investigation, it was not anticipated that the Be-shell stars would have low surface gravities. Yet the values of $\log g$ found in this investigation for these objects range from 3.2-3.5. Although the reason for the low gravities is not immediately evident, we can offer the following three suggestions.

(1) The stars may simply be in the early stages of their post main sequence, hydrogen shell burning phase. If this is the case, then it is not clear why they should have emission line envelopes and 'metallic shells'. It should be mentioned that $28 \mathrm{Tau}$, a well-known member of the Pleiades, appears to be located near the main sequence (Johnson and Mitchell 1958).

(2) Shell stars could have extremely high rotational velocities and, hence, low mean surface gravities. However, rotational velocities in excess of $90 \%$ of the critical value are required to reduce the mean $\log g$ to the range of values obtained in this investigation. For example, if $v_{\mathrm{eq}}=350 \mathrm{~km} \mathrm{~s}^{-1}$ and $\log g_{p}=3.9$, then $\log g_{\mathrm{eq}}=3.7$. The mean $\log g$ for such a star is 3.8.

(3) The model atmospheres which were used for the analysis may not be representative of the photospheres of shell stars. However, at this time it is not possible to decide appropriateness of the models since we are as yet uncertain about the rotational velocities and surface geometries of shell stars.

It is my feeling that there are several mechanisms for the formation of $\mathrm{Be}$ envelopes. This investigation has shown that the surface gravities for Be stars are comparable to those determined for non-Be stars. The Be stars apparently do not display a narrow range in $\log g$. Actually, the wide range in luminosity classes (III-V) which have been assigned to Be stars allows one to anticipate this conclusion. On the other hand, the Be-shell stars seem to have low surface gravities. These objects invariably are classified as Bp! Perhaps by making use of new observations in several spectral regions combined with new line transfer computations for circumstellar envelopes we can eventually isolate the various groups of $\mathrm{Be}$ stars.

\section{Acknowledgements}

I wish to thank Dr M. Plavec for the support that he has given this project in the form of generous amounts of his time on the Lick Observatory 120-in. telescope. The project was part of an extensive program at UCLA aimed at studying interacting binary systems and was supported in part by NSF MPS 74-04194A01 (Popper/Plavec). I also wish to acknowledge numerous interesting and helpful discussions with Dr M. Plavec and R. S. Polidan. 


\section{References}

Blanco, V. M., Demers, S., Douglass, G. G., and Fitzgerald, M. P.: 1968, Publ. U.S. Naval Obs. 21.

Bodenheimer, P. and Ostriker, J. P.: 1973, Astrophys. J. 180, 159.

Burbidge, G. R. and Burbidge, E. M.: 1953, Astrophys. J. 117, 407.

Crampin, J. and Hoyle, F.: 1960, Monthly Notices Roy. Astron. Soc. 120, 33.

Feinstein, A.: 1974, Monthly Notices Roy. Astron. Soc. 169, 171.

Heap, S. R.: 1975, personal communication.

Johnson, H. L.: 1966, Ann. Rev. Astron. Astrophys. 4, 193.

Johnson, H. L. and Mitchell, R. I.: 1958, Astrophys. J. 128, 31.

Kodaira, K. and Scholz, M.: 1970, Astron. Astrophys. 6, 93.

Koubsky, P.: 1975, IAU Circ. No. 2802.

Kriz, S. and Harmanec, P.: 1975, Bull. Astron. Inst. Czech. 26, 65.

Lesh, J. R.: 1968, Astrophys. J. Suppl. 17, 371.

Limber, D. N.: 1970, in A. Slettebak (ed.), Stellar Rotation, D. Reidel Publ. Corp., Dordrecht-Holland, p. 274.

Limber, D. N. and Marlborough, J. M.: 1968, Astrophys. J. 152, 181.

Peters, G. J.: 1972, Publ. Astron. Soc. Pacific 84, 334.

Peters, G. J.: 1974, Bull. Am. Astron. Soc. 6, 456.

Peters, G. J.: 1976, in preparation.

Peters, G. J. and Polidan, R. S.: 1973, in A. H. Batten (ed.), 'Extended Atmospheres and Circumstellar Matter in Spectroscopic Binary Systems', IAU Symp. 51, p. 174.

Peters, G. J. and Polidan, R. S.: 1975, IAU Circ. No. 2814.

Plavec, M.: 1970, Publ. Astron. Soc. Pacific 82, 957.

Plavec, M.: 1973, in A. H. Batten (ed.), 'Extended Atmospheres and Circumstellar Matter in Spectroscopic Binary Systems', IAU Symp. 51, p. 216.

Sackmann, I. J. and Anand, S. P. S.: 1970, Astrophys. J. 162, 105.

Schild, R.: 1973, Astrophys. J. 179, 221.

Schild, R., Chaffee, F., Frogel, J. A., and Persson, S. E.: 1974, Astrophys. J. 190, 73

Slettebak, A.: 1952, Astrophys. J. 115, 573.

Uesugi, A. and Fukuda, I.: 1970, Contrib. Inst. Astrophys. Kwasan Obs. Univ. Kyoto, No. 189.

Vidal, C. R., Cooper, J., and Smith, E. W.: 1973, Astrophys. J. Suppl. 25, 37. 